\title{
Validación de un video educativo para fortalecer las prácticas de parto humanizado entre personal de salud
}

\author{
Validation of an educational video to strengthen humanized childbirth practices \\ among healthcare workers
}

\author{
Validação de um vídeo educativo para fortalecer as práticas do parto humanizado \\ entre pessoal da área de saúde
}

\author{
Giselly Mayerly Nieves Cuervo; Angie Daniela Lizarazo-Castellanos²; Flor de María Cáceres-Manrique ${ }^{3}$ \\ 1 Especialista en Gerencia Integral de Servicios de Salud. Universidad Industrial de Santander, Santander, Colombia. giselly_mayi20@ \\ hotmail.com. ORCID: https://orcid.org/0000-0003-1893-2765 \\ 2 Médica y cirujana. Universidad Industrial de Santander, Santander, Colombia. angie_dani25@hotmail.com. ORCID: https://orcid. \\ org/0000-0002-5201-9112 \\ 3 Doctora en Salud Pública. Universidad Industrial de Santander, Santander, Colombia. fmcacer@uis.edu.co. ORCID: https://orcid. \\ org/0000-0001-7058-5815
}

Recibido: 26/11/2020. Aprobado: 02/09/2021 Publicado: 30/11/2021

\begin{abstract}
Nieves-Cuervo GM, Lizarazo-Castellanos AD, Cáceres-Manrique FM. Validación de un video educativo para fortalecer las prácticas de parto humanizado entre personal de salud. Rev. Fac. Nac. Salud Pública. 2022; 40(1):e344413. DOI: https://doi. org/10.17533/udea.rfnsp.e344413
\end{abstract}

\section{Resumen}

Objetivo: Validar un video educativo sobre parto humanizado, dirigido al personal de salud, con el fin de fortalecer e incentivar prácticas en salud pública que aseguren el respeto de los derechos de las maternas y promuevan el bienestar del binomio madre-hijo. Metodología: Validación de tecnología educativa en formato video. Se realizó una búsqueda bibliográfica y una selección de contenidos. También se elaboró el libreto y se produjo un video, que fue validado por 6 especialistas, seleccionados según su hoja de vida, y por 222 profesionales y técnicos del área de salud, quienes atienden gestantes y maternas. Los evaluadores fueron contactados por redes sociales, usando muestreo en "bola de nieve". Se calculó el índice de validez de contenido, considerado aceptable cuando es mayor que 0,60. Resultados: Un video que expone, en 2 minutos y 20 segundos, los derechos de las gestantes y maternas, y motiva su cumplimiento. En su validación participaron 6 especialistas, 4 con título de maestría y 2 con doctorado, quienes calificaron el índice de validez de contenido con máxima puntuación. La población objetivo, principalmente médicos y enfermeras, asignaron el índice de 
validez de contenido entre 0,92 y 0,99 , con una concordancia de 97,87. Ambos grupos hicieron comentarios positivos y dieron su aprobación al video. Conclusión: Se obtuvo un video educomunicativo corto, calificado con altos índices de validez por especialistas y público objetivo, quienes lo consideraron útil para fortalecer las prácticas de parto humanizado entre el personal de salud e incentivar el respeto de los derechos de las maternas.

Palabras clave: Colombia, educomunicación, educación en salud, estudios de validación, parto humanizado, recursos audiovisuales.

\section{Abstract}

Objective: To validate an educational video about humanized childbirth, aimed at healthcare workers, in order to strengthen and encourage public health practices that ensure respect for the rights of mothers and promote the well-being of the mother-infant dyad. Methodology: Validation of educational technology in video format. A bibliographic search and a selection of contents were carried out. A script was created and a video was produced, which was validated by 6 specialists, selected according to their resume, and by 222 health professionals who work with pregnant women. Evaluators were contacted by social networks, using a "snowball " sampling. The content validity index was calculated, and was considered acceptable when greater than 0.60. Results: A 2 minutes and 20 seconds video presented the rights of pregnant women and mothers in order to motivate their compliance to treatments. For video validation, 6 specialists participated, 4 hold a master's degree and 2 hold a doctorate, who rated the content validity index with the highest score. The target population, mostly doctors and nurses, assigned the content validity index between 0.92 and 0.99 , with a concordance of 97.87 . Both groups made positive comments and approved the video. Conclusion: A short educational video was obtained, qualified with high validity rates by specialists and target audience, who considered it useful to strengthen humanized childbirth practices among healthcare workers and encourage respect for the rights of mothers.

-Keywords: Colombia, educommunication, health education, validation studies, humanized childbirth, audiovisual resources.

\section{Resumo}

Objetivo: Validar um vídeo educativo sobre parto humanizado, dirigido ao pessoal da área de saúde, com o objetivo de fortalecer e incentivar as práticas em saúde pública que possam garantir o respeito aos direitos das gestantes e que promova o bem-estar do binômio mãe-filho. Metodologia: Validação da tecnologia educacional em formato de vídeo. Foram realizadas uma pesquisa bibliográfica e uma seleção de conteúdos. Além disso, foi elaborado um texto para a produção do vídeo, validado por 6 especialistas, selecionados de acordo a seus Curricula Vitae e por 222 profissionais e técnicos da área de saúde, os quais atendem a gestantes e puérperas. Os avaliadores foram contatados pelas redes sociais, usando amostragem em "bola de neve". Foi calculado o índice de validade de conteúdo e se considera aceitável quando seja superior a 0,60. Resultados: Um vídeo que expõe, em 2 minutos e 20 segundos, os direitos das gestantes e puérperas e motiva seu cumprimento. Em sua validação participaram 6 especialistas, 4 com títulos de mestrado e 2 com doutorado, os quais deram a nota máxima ao índice de validade de conteúdo. O público-alvo, principalmente médicos e enfermeiras, designou o índice de validade do conteúdo entre 0,92 e 0,99 com uma concordância de 97,87. Ambos os grupos fizeram comentários positivos e deram sua aprovação ao vídeo. Conclusão: Foi obtido um vídeo educomunicativo curto, qualificado com altos índices de validade por especialistas e público-alvo, que o consideraram útil para fortalecer as práticas de parto humanizado entre o pessoal da área de saúde e incentivar o respeito aos direitos das gestantes.

----------Palavras-chave: Colômbia; Educomunicação; Educação em saúde; Estudos de validação; Parto humanizado; Recursos audiovisuais 


\section{Introducción}

El parto humanizado es un constructo compuesto por atributos [1] tendientes a garantizar el derecho de las mujeres a vivir la gestación, el trabajo de parto, el parto y el posparto con libertad de decisión, consciencia y respeto. Recomienda llevar a cabo un mínimo de intervenciones necesarias para garantizar el bienestar de la madre y el hijo, con el propósito de evitar la violencia obstétrica, que se manifiesta cuando estos atributos no se cumplen, que está enmarcada entre las violencias de género [2-3], y que es una preocupación para la salud pública, dado que se produce en las instituciones, por motivo o con ocasión de la atención a las gestantes.

La humanización de la atención es objeto de estudio reciente, que busca ubicar a la mujer en el centro de atención, como protagonista del cuidado, en pro de garantizar el respeto de sus derechos humanos, sexuales y reproductivos, así como el derecho a la intimidad, la integridad personal, la privacidad, la autonomía, la dignidad, y a no ser sometida a tratos crueles $[4,5]$. La humanización de la atención en los servicios de salud es también de interés para la salud pública, dado que suele opacarse por la tecnificación del cuidado, el afán por la eficiencia, el control de costos y la rentabilidad financiera [6].

La garantía de humanización parte del conocimiento que al respecto tenga el personal de salud sobre el tema, de su entrenamiento y de la convicción para propiciar escenarios humanizados, donde dicho personal funge como mediador de vínculos y es responsable de armonizar las tecnologías y el conocimiento con el buen trato, en procura del bienestar de las maternas.

Para incentivar el conocimiento sobre humanización, se requiere contar con estrategias pedagógicas masivas, mediadas por tecnologías de la comunicación y la educación (TIC), que permitan educar, optimizando el tiempo, y aprovechar la combinación de conocimientos, información, imágenes, sonido y texto, en un mundo de redes sociales que facilitan la interconexión del cuidado de la salud, con los campos de estudio de la educación y la comunicación [7], para reforzar los saberes y motivar la humanización de la atención. Sin embargo, son escasas dichas tecnologías y muy pocas han sido validadas [8].

La educomunicación vislumbra un panorama de oportunidades y avances científicos en salud, con el advenimiento de las TIC [7]. Es concebida como la disciplina que estudia la relación entre comunicación y educación, para optimizar recursos en el proceso de enseñanza-aprendizaje, al armonizar medios de comunicación y procesos comunitarios [9]. Así, permite generar cambios en las percepciones y conductas del personal de salud, acordes con el contenido y el impacto esperado [10], mediante el uso de recursos físicos y humanos, me- dios audiovisuales, plataformas digitales, folletos y actividades lúdicas interactivas, entre otros [11]. Los medios audiovisuales contribuyen a la educación en salud, mostrando efectividad en la difusión y la capacitación, en la replicación de la información [12], así como en el desarrollo de competencias clínicas, al aproximar a los participantes a la práctica en escenarios reales [13] y a la reflexión sobre sus acciones para el cuidado de la salud.

Ahora bien, los videos han tomado auge como recursos educativos en diferentes áreas, dado que permiten ilustrar temas científicos, involucrar a los participantes, incentivar la humanización de la atención [14] y contribuyen al desarrollo de las mejores prácticas en salud [13]. Se consolidan como herramientas didácticas en salud pública, por su capacidad para lograr impacto en las creencias y conductas de las personas, y por su utilidad para fortalecer el conocimiento médico [15]. Son una de las tecnologías de innovación educativa que permite mayor difusión de información en el menor tiempo posible, al generar entornos agradables de aprendizaje [16]. Lo anterior, sumado al poder de divulgación que tienen las redes sociales, es una motivación para crear un video con contenido válido, con evidencia científica, que sea útil para mejorar la calidad de los servicios de salud y la humanización del cuidado [17], al mismo tiempo que permita aclarar la desinformación creciente en las redes sociales [18] e impactar en la salud pública.

En este escenario, se requiere elaborar y validar estrategias educomunicativas sobre parto humanizado, con especialistas y personal de salud, para lograr adecuados niveles de entendimiento y comprensión del tema, inducir a la acción, orientar el comportamiento a partir de la información sobre qué es humanización y cómo realizarla [19]. En ese sentido, este estudio tuvo como objetivo validar un video educativo sobre parto humanizado, dirigido al personal de salud, con el fin de fortalecer e incentivar prácticas en salud pública que aseguren el respeto de los derechos de las maternas y promuevan el bienestar del binomio madre-hijo.

\section{Metodología}

Se elaboró y validó una tecnología educativa en formato de video, sobre el parto humanizado. El video está dirigido al personal de salud que atiende a la mujer gestante durante el embarazo, el trabajo de parto, el parto o el puerperio. La elaboración y la validación se llevaron a cabo en cinco etapas, siguiendo la propuesta de un estudio previo [14]:

1. Búsqueda de los contenidos. Consistió en la búsqueda y la revisión bibliográfica sobre parto humanizado, humanización de la atención, prevención de la violencia obstétrica y humanización del cuidado de las gestantes. La búsqueda se realizó 
en las bases de datos Literatura Latinoamericana y del Caribe en Ciencias de la Salud (LILACS), la Scientific Electronic Library Online (SciELO) y PubMed $\AA$, y fue complementada con la revisión de los lineamientos y las guías nacionales [20] e internacionales [21] sobre parto humanizado. De la literatura revisada, se seleccionaron, por consenso de los investigadores, los contenidos considerados pertinentes para cumplir con el objetivo educativo del video. Dichos contenidos se organizaron por temas, para desarrollar las escenas del video, así: a) definición de parto humanizado, b) objetivos, c) responsabilidades del personal de salud, d) derechos de la materna, e) hallazgos preliminares, no publicados, de una investigación multicéntrica sobre el tema en Colombia, ${ }^{*}$ y f) reflexiones finales e invitación a propiciar atención humanizada a gestantes y maternas.

2. Elaboración del libreto y producción del video. Una vez definido el contenido y los temas, se elaboró un libreto, con la secuencia de escenas en el orden establecido, siguiendo el modelo de flujo propuesto en estudios previos [22-23]. Cada escena describía el texto y la forma a ser narrado: oral o escrita, acompañado de imágenes, movimiento, cambios de tono de voz, silencios, lenguaje oral, visual, sonidos y colores. Todo ello con el fin de tener una visión inicial de cómo quedaría representada cada escena en el video. Con estas especificaciones, se acudió a los servicios de un equipo de producción, compuesto por diseñadores y locutores, quienes produjeron versiones sucesivas del video, hasta lograr un producto armónico, con el cual estuvieron de acuerdo tanto investigadores como productores de la tecnología audiovisual.
3. Validación del video por parte de los expertos. Para dar cumplimiento a este paso, se determinó presentarlo a expertos o especialistas en el tema, profesionales con trayectoria en atención clínica, docencia o investigación, bien fuera en humanización de los servicios, salud materna, cuidado perinatal o educación para la salud. Así, se elaboró un listado de 25 potenciales expertos y se buscó información detallada de sus hojas de vida, con el fin de verificar si cumplían los criterios de inclusión. Para ser clasificados como expertos, se estableció que se requería obtener, a partir de la evaluación de su hoja de vida, al menos diez puntos, de acuerdo con la escala de criterios adaptada de otros autores [24] a los objetivos del presente estudio (véase Tabla 1).

De los 25 potenciales expertos, 6 cumplieron los criterios de selección y obtuvieron calificación de 10 puntos o más, según los méritos académicos reportados en sus hojas de vida. Ellos fueron contactados por correo electrónico, dieron su consentimiento informado para participar en el estudio, revisaron el video e hicieron la correspondiente calificación en el formato elaborado para tal fin, descrito en el siguiente apartado.

4. Validación del video por parte de la población objetivo. La población a la cual va dirigido el video o población objetivo, corresponde al personal de salud que tiene a su cargo el cuidado de las maternas en cualquier momento de gestación.

Esta población fue convocada por las redes sociales, para obtener valoración de la compresión de los objetivos del video, la importancia/relevancia de este como material educativo y su conformidad con la apariencia de la estrategia presentada.

Para garantizar la participación de personal de salud de diferentes categorías y niveles de formación,

Tabla 1. Criterios de cualificación para ser considerado experto

\begin{tabular}{lc}
\hline & Ítem calificable en hoja de vida de expertos \\
\hline Título de doctorado & 4 \\
Título de maestría & 3 \\
Publicación en revista indexada o producción bibliográfica sobre parto humanizado & 2 \\
Especialización en área de interés del estudio: educomunicación/parto & 2 \\
Experiencia laboral de mínimo 5 años en cuidado materno/perinatal & 2 \\
Participación en evento científico en los últimos dos años sobre atención materna/perinatal o humanización de la \\
atención
\end{tabular}

Fuente: adaptado de Fehring [24].

* "Prevalencia y factores asociados al parto humanizado en siete ciudades de Colombia, 2018". Investigación multicéntrica, en curso, cofinanciada por Colciencias (Código 130880763149) y seis universidades del país. 
se convocaron técnicos, tecnólogos, profesionales, especialistas clínicos, especialistas no clínicos, profesionales con maestría, doctorado o posdoctorado. Se contactaron por redes sociales por muestreo no probabilístico, intencional, utilizando estrategia de bola de nieve.

Además, se envió un formulario electrónico elaborado en la plataforma de Google Forms, en línea, organizado por secciones: en una primera sección se presentaban los objetivos de la investigación y se solicitaba el consentimiento informado para participar; en la segunda se insertaba el video mediante un enlace, para que fuera visualizado por el participante; en la tercera sección se presentaba el formato con las preguntas para la calificación, y en la última se solicitaba información personal del participante, como edad, sexo y formación académica.

En cuanto al instrumento para la calificación del video, corresponde a la adaptación de un formato previamente diseñado [14]. El formato final estuvo conformado por 26 preguntas, orientadas a evaluar si el video cumple con los atributos esperados en cuanto a: 1) objetivos, mediante seis preguntas, 2) estructura y presentación, 12 preguntas, y 3) relevancia del video como tecnología educomunicativa, mediante 8 preguntas. La calificación de cada pregunta se hizo en escala tipo Likert, donde el participante señalaba en cada pregunta si estaba en desacuerdo, parcialmente de acuerdo o de acuerdo.

Para calcular el índice de validez de contenido (IVC), se sumaron las respuestas positivas, es decir, las calificadas como de acuerdo o parcialmente de acuerdo, en relación con el puntaje máximo posible, si todos los evaluadores juzgaran todos los ítems de manera positiva. Así:

$$
I V C=(n-N / 2) /(N / 2)
$$

donde $n$ representa el número de personas que califican el ítem y $N$ el número total de evaluadores.

Se asumió como aceptable un IVC mayor que 0,60 y se reevaluaron aquellos ítems cuyo IVC estuvo por debajo de este valor [24].

La concordancia se calculó mediante el índice kappa $(\kappa)$, con la siguiente fórmula:

$$
\kappa=(P o-P e) /(1-P e),
$$

donde $P o$ es la proporción de concordancia observada y $P e$ la concordancia esperada [25].

Se asumió aceptable una $\kappa$ mayor que 0,80 .

Además de las preguntas, el formato ofrecía un espacio destinado para que los participantes consignaran inquietudes, opiniones y sugerencias adicionales.

Las respuestas a los formatos fueron automáticamente sistematizadas y recuperadas en bases de datos en el programa estadístico Stata $14^{\circledR}$ (StataCorp. 2015, College Station, licencia de la de la Universidad Industrial de Santander, Departamento de Salud Pública) para efectuar el análisis de la información, el cual consistió en el cálculo del IVC, el $\kappa$ y la descripción de los participantes, según edad, sexo y nivel educativo.
La quinta y última etapa fue la adecuación del video según los hallazgos. Se hicieron los ajustes sugeridos por los evaluadores, se modificaron algunas imágenes, se dieron los créditos a las instituciones participantes y se publicó el video en las redes sociales más usadas por la población general: YouTube, Facebook y WhatsApp.

Para el estudio se siguieron los lineamientos éticos de la Resolución 8430 de 1993 del Ministerio Salud de Colombia [26], según la cual se clasifica como investigación con riesgo mínimo.

La investigación en la que anida el estudio fue avalada por el Comité de Ética de la Universidad El Bosque, de Bogotá, según Acta 012-2018 del 5 de mayo de 2018.

\section{Resultados}

Se obtuvo un video cuya versión final tiene una duración de 2 minutos y 19 segundos. Este muestra los aspectos más importantes a tener en cuenta en la atención de las gestantes durante el embarazo, parto y posparto, y tiene como propósitos pedagógicos educar, informar y comunicar estrategias de parto humanizado al personal de salud que atiende gestantes y maternas, e incentivar en él el ejercicio de esta práctica.

La versión sin ajustes se puede ver en: https:// www.youtube.com/watch? $v=S T z F z 1 Y F D N o \& t=9 s$. La versión con los arreglos sugeridos por los evaluadores se encuentra disponible en: https://youtu.be/ cMy6m5SPBqQ.

Los expertos que participaron en la validación del video fueron 3 hombres y 3 mujeres. Uno de ellos obtuvo 11 puntos de calificación según su hoja de vida; los 5 restantes alcanzaron puntajes de 10 . Sus edades estaban entre los 38 y 65 años, con media de 40 años. En formación profesional, 4 eran enfermeros $(66,66 \%)$, un médico y una nutricionista; 4 tenían título de maestría $(66,67 \%)$ y 2 de doctorado (33,33\%). Estos especialistas calificaron la validez de contenido (IVC) del video con máxima nota, es decir, dieron un puntaje de 1, con un índice de concordancia $(\kappa)$ del $100 \%$. Solo hubo 4 respuestas de "parcialmente de acuerdo" en preguntas diferentes del formato de evaluación.

Por su parte, la población objetivo estuvo compuesta por 222 personas, quienes evaluaron el video y diligenciaron el instrumento. Fueron 140 mujeres $(63,06 \%)$ y 82 hombres (36,94\%), con edades entre 20 y 63 años, promedio de 40 años, la mayoría entre 25 y 29 años $(17,57 \%)$. Su formación era así: 80 médicos $(36,04 \%)$, 75 enfermeros ( $33,78 \%)$ y 67 eran otros profesionales y técnicos del área de la salud (30,18\%), incluyendo dos doulas; 69 participantes $(31,08 \%)$ contaban con formación de pregrado, y 43, con posgrado clínico (19,37\%). Una persona tenía posdoctorado. 
Las características de la población objetivo se presentan en la Tabla 2.

La población objetivo calificó las tres secciones del video con un IVC que osciló entre 0,92 y 0,99 , con una concordancia media de $97,87 \%$ ( $\kappa$ entre 96,40 y 99,55 $\%)$. La pregunta con más profesionales en desacuerdo fue si el tipo de estrategia educativa (video) es apropiada para personal de salud $[n=10(4,50 \%)]$, seguida de si el video permite la sensibilización del personal para la humanización de la atención en salud [ $n=8(3,60 \%)]$, y si el material permite la generalización del aprendizaje a los diferentes servicios de atención de la materna $[n=8$ $(3,60 \%)]$. Sin embargo, estos desacuerdos corresponden a menos del $5 \%$ y no son estadísticamente significativos.

En la Tabla 3 se muestra la calificación asignada por los participantes a cada una de las preguntas del formato.

Tabla 2. Características de los trabajadores de la salud, participantes en la validación del video

\begin{tabular}{llcc}
\hline Característica de los participantes & Número de participantes (222) & \% \\
\hline $20-24$ & 18 & 8,11 \\
$25-29$ & 39 & 17,57 \\
Edad (años) & $35-34$ & 32 & 14,41 \\
& $40-44$ & 25 & 11,26 \\
& $45-49$ & 28 & 12,61 \\
& $50-54$ & 21 & 9,46 \\
& $55-59$ & 16 & 7,21 \\
& $60+$ & 22 & 9,91 \\
Formación profesional & Médico & 21 & 9,46 \\
\hline & Enfermero & 80 & 36,04 \\
& Auxiliar de enfermería & 75 & 33,78 \\
& Psicólogo & 10 & 4,50 \\
& Odontólogo & 8 & 3,60 \\
& Fisioterapeuta & 5 & 2,25 \\
& Nutricionista & 3 & 1,35 \\
& Bacteriólogo & 3 & 1,35 \\
& Otro & 2 & 0,90 \\
Nivel de formación alcanzado & 36 & 16,22 \\
\hline & Pregrado profesional & 69 & 31,08 \\
& Posgrado clínico & 43 & 19,37 \\
& Posgrado no clínico & 40 & 18,02 \\
& Técnico/tecnológico & 40 & 18,02 \\
& Doctorado & 9,01 \\
Posdoctorado & 1 & 4,05 \\
& & 0,45 \\
\hline
\end{tabular}

Tabla 3. Índice de validez de contenido por cada ítem del instrumento de validación del video según calificación dada por la población objetivo

\begin{tabular}{|c|c|c|c|c|c|}
\hline Contenido & Preguntas de validación & $\begin{array}{c}\text { En } \\
\text { desacuerdo }\end{array}$ & $\begin{array}{c}\text { Parcialmente } \\
\text { de acuerdo }\end{array}$ & $\begin{array}{c}\text { De } \\
\text { acuerdo }\end{array}$ & $\operatorname{IVC}^{\star \star}$ \\
\hline \multirow{6}{*}{ Objetivo } & $\begin{array}{l}\text { ¿La información es coherente con los lineamientos del parto } \\
\text { humanizado? }\end{array}$ & 1 & 29 & 192 & 0,99 \\
\hline & ¿El objetivo es claro? & 2 & 23 & 197 & 0,98 \\
\hline & ¿La información es relevante para la humanización del parto? & 4 & 34 & 184 & 0,96 \\
\hline & ¿El video invita a realizar cambios comportamentales? & 6 & 51 & 165 & 0,94 \\
\hline & ¿El video es coherente con el área científica? & 5 & 33 & 184 & 0,95 \\
\hline & ¿Atiende al objetivo de educar sobre parto humanizado? & 4 & 30 & 188 & 0,96 \\
\hline
\end{tabular}




\begin{tabular}{|c|c|c|c|c|c|}
\hline Contenido & Preguntas de validación & $\begin{array}{c}\text { En } \\
\text { desacuerdo }\end{array}$ & $\begin{array}{l}\text { Parcialmente } \\
\text { de acuerdo }\end{array}$ & $\begin{array}{c}\text { De } \\
\text { acuerdo }\end{array}$ & $\operatorname{IVC}^{\star *}$ \\
\hline \multirow{12}{*}{$\begin{array}{l}\text { Estructura y } \\
\text { presentación }\end{array}$} & $\begin{array}{l}\text { ¿El tipo de estrategia educativa (video) es apropiada para } \\
\text { personal de salud? }\end{array}$ & 10 & 38 & 174 & 0,99 \\
\hline & ¿El mensaje es claro? & 2 & 30 & 190 & 0,98 \\
\hline & ¿El video utiliza el lenguaje científico correcto? & 3 & 27 & 192 & 0,97 \\
\hline & $\begin{array}{l}\text { ¿El contenido es apropiado al nivel sociocultural del personal } \\
\text { de salud? }\end{array}$ & 3 & 35 & 184 & 0,97 \\
\hline & ¿Hay secuencia lógica de presentación? & 7 & 25 & 190 & 0,93 \\
\hline & $\begin{array}{l}\text { ¿Hay armonía entre el texto, las figuras y la voz para trasmitir } \\
\text { el mensaje? }\end{array}$ & 2 & 21 & 199 & 0,98 \\
\hline & $\begin{array}{l}\text { ¿El estilo de redacción corresponde al nivel de conocimiento } \\
\text { del público objetivo? }\end{array}$ & 4 & 30 & 188 & 0,96 \\
\hline & ¿El tamaño de la letra es adecuado? & 2 & 12 & 208 & 0,98 \\
\hline & ¿Las ilustraciones son expresivas? & 6 & 19 & 197 & 0,94 \\
\hline & ¿El audio es apropiado? & 3 & 4 & 215 & 0,97 \\
\hline & ¿La duración del video es apropiada? & 2 & 21 & 199 & 0,98 \\
\hline & ¿El material visual es apropiado? & 3 & 22 & 197 & 0,97 \\
\hline \multirow{8}{*}{ Relevancia } & $\begin{array}{l}\text { ¿El video enfatiza en aspectos claves que deben ser } \\
\text { reforzados? }\end{array}$ & 6 & 60 & 156 & 0,95 \\
\hline & ¿El video aborda un tema primordial de salud pública? & 6 & 13 & 203 & 0,95 \\
\hline & $\begin{array}{l}\text { ¿El material permite la generalización del aprendizaje a los } \\
\text { diferentes servicios de atención de la materna? }\end{array}$ & 8 & 39 & 175 & 0,93 \\
\hline & ¿El video propone la aplicación del conocimiento? & 6 & 40 & 176 & 0,95 \\
\hline & $\begin{array}{l}\text { ¿El video expone los conocimientos que el personal de salud } \\
\text { debe tener sobre parto humanizado? }\end{array}$ & 6 & 60 & 156 & 0,95 \\
\hline & $\begin{array}{l}\text { ¿El video permite reforzar el conocimiento sobre parto } \\
\text { humanizado en el personal de salud? }\end{array}$ & 7 & 45 & 170 & 0,94 \\
\hline & $\begin{array}{l}\text { ¿El video permite la sensibilización del personal para la } \\
\text { humanización de la atención en salud? }\end{array}$ & 8 & 45 & 169 & 0,93 \\
\hline & ¿El video es adecuado para cualquier profesional de salud? & 7 & 21 & 194 & 0,94 \\
\hline
\end{tabular}

$n$ : número de participantes; IVC: Índice de validez de contenido.

\section{Discusión}

Este estudio permitió elaborar y validar un video educomunicativo, dirigido al personal de salud, a partir de la revisión bibliográfica [1-7] y del seguimiento de los lineamientos para la realización y validación de recursos audiovisuales [12-14]. Tanto los expertos como el personal de salud otorgaron altos índices de IVC y lo evaluaron como adecuado para brindar educación e invitar a adoptar conductas de atención humanizada durante la gestación, el parto y el posparto.

Este tipo de estrategias ha mostrado influir en el cambio de percepción y comportamiento, que se ve reflejado en el desarrollo de mejores prácticas en salud [15-17] y como recurso para capacitación y autoaprendizaje [12], lo cual facilitaría la adherencia del personal de salud a la humanización de la atención brindada a las maternas [6], así como la replicación y la difusión de la información, en un corto tiempo.

Los resultados obtenidos en el presente estudio son similares a una investigación previa, que validó un video educativo para la enseñanza de la reanimación cardiopulmonar a estudiantes sordos, cuyos resultados de concordancia también fueron altos $(80 \%)$ y las discrepancias mínimas [12]. Ya en el tema específico, un estudio previo validó una cartilla con el propósito de educar a gestantes y familiares sobre parto humanizado. Siguiendo una ruta metodológica similar, llegó a un ejemplar con IVC y confiabilidad adecuados para dar educación a maternas y población general sobre el tema [8]. Se espera, con estos hallazgos, contar con herramientas para difundir el conocimiento y motivar hacia la humanización en la atención a las maternas; pero se requiere seguir alimentando la línea de investigación, para lograr impactar la atención del binomio madre-hijo y el bienestar de la familia como apoyo a las políticas de salud pública.

Entre las fortalezas de este estudio están: el soporte de literatura y la realización de un proceso sistemático de selección de contenidos, imágenes, colores y voces, acordes con el mensaje a difundir en el video [16]; la combinación armónica de los anteriores elementos, para lograr un video corto, pero con los atributos necesarios para di- 
fundir el mensaje; la posterior validación del video, previo establecimiento de criterios de inclusión y selección cuidadosa de los expertos [14, 24], así como la participación de un número considerable de personal de salud, de diferentes profesiones y grados de formación académica.

Además, se destaca la corta duración del video y las potenciales formas de difusión, haciendo uso de las TIC, a través de las redes sociales de amplio cubrimiento, como YouTube, Facebook y WhatsApp, con acceso gratuito y alta difusión, a bajo costo, en un escenario donde el tiempo es muy valioso, con escasos espacios para la reflexión sobre la humanización del cuidado de salud y el respeto por los derechos de las maternas.

A su vez, se perciben varias debilidades, como el hecho de que solo 6 de los 25 especialistas convocados cumplieron con los criterios de inclusión para participar en la validación del video; sin embargo, esto también habla de la exigencia de los criterios con relación a la competencia académica, laboral e investigativa, de quienes participaron en el proceso.

Por otro lado, la complejidad misma del tema y el reciente reconocimiento como problema de salud pública hacen que los estudios sean escasos, como también lo son las posibilidades de comparación de los resultados con estudios sobre dicho tema y diseño metodológico.

Además, la falta de familiaridad y poco reconocimiento sobre la importancia del parto humanizado, así como el hecho de que este no solo depende de la conducta y de los conocimientos de los trabajadores de la salud, sino también de otros factores, como la organización y la dotación de los servicios y de la disposición de espacios adecuados para brindar una atención humanizada $[1,20$, 21], advierten que las acciones de mejora dependen tanto de los actuales resultados como de los esfuerzos adicionales del sector salud [27].

Esta investigación aporta una tecnología válida para educar al personal, pero los otros aspectos deberán ser abordados desde la administración de los servicios de atención a las maternas.

Cabe anotar que existe una delgada línea entre la percepción de la violencia obstétrica y la falta de atención humanizada de las maternas [1-5]. Se carece de orientación clara y expedita en la promoción del parto humanizado y todo lo que este proceso implica, lo cual genera incumplimiento del respeto por los derechos de la mujer $[3,28]$.

La humanización de la atención es una prioridad de salud pública, una oportunidad para los trabajadores de la salud, una necesidad para las maternas y sus familias, quienes tienen el derecho de ser protagonista de su cuidado y ser atendida en forma humanizada; sin embargo, se requiere más investigación, capacitación profesional e infraestructura física, para promover atención humanizada [29]. Se pretende incidir en los profesionales de salud con acceso a redes sociales de cualquier parte del mundo, mediante procesos creativos de educomunicación [7,9-11], que favorezcan la humanización de la atención a partir de estrategias válidas y de fácil difusión.

\section{Conclusión}

Se produjo un video válido como estrategia educomunicativa sobre parto humanizado, dirigida al personal de salud que atiende gestantes en cualquier etapa del proceso de gestación. Es una estrategia novedosa, corta, fácil de compartir, y se espera que genere nuevas investigaciones y estrategias de educación, información y comunicación para ayudar al personal de salud a fomentar la atención humanizada, en aras de conseguir una atención con calidez y calidad.

\section{Agradecimiento}

A Colciencias (actual MinCiencias) y a la Universidad Industrial de Santander por la cofinanciación del proyecto y del Programa Jóvenes Investigadores.

\section{Conflicto de interés}

Las autoras no tenemos conflicto de intereses.

\section{Fuente de financiación}

La Universidad Industrial de Santander financió a Flor de María Cáceres-Manrique como investigadora, y cofinanció las becas, junto con Colciencias, a través del Programa Jóvenes Investigadores 2018 Código 809 para Giselly Mayerly Nieves Cuervo y Angie Daniela Lizarazo-Castellano.

\section{Declaración de responsabilidad}

El contenido del artículo es responsabilidad únicamente de las autoras.

\section{Declaración de autoría}

Las autoras (Flor de María Cáceres-Manrique, Giselly Mayerly Nieves Cuervo y Angie Daniela Lizarazo-Castellano) participaron en la concepción y el diseño del estudio; en la revisión de la literatura para elaboración del video, y en la recolección, el análisis e interpretación de los datos. Giselly Mayerly Nieves Cuervo y Angie Daniela Lizarazo-Castellano elaboraron el primer borrador de la "Introducción" y el "Método" del artículo. Flor de María Cáceres-Manrique se encargó de la escritura 
inicial de los "Resultados" y la "Discusión". Todas revisamos y armonizamos la versión enviada a publicación. Todas estamos de acuerdo con la versión final del documento.

\section{Referencias}

1. Curtin M, Savage E, Leahy-Warren P. Humanisation in pregnancy and childbirth: A concept analysis. J Clin Nurs. 2020;29:1744-57. DOI: http://dx.doi.org/10.1111/jocn. 15152

2. Barbosa-Mendes R, Santos JMJ, Siqueira-Prado D, et al. Evaluation of the quality of prenatal care based on the recommendations Prenatal and Birth Humanization Program. Ciênc. Saúde Coletiva, 2020;25(3):793-804. Dor: https://doi.org/10.1590/141381232020253.13182018

3. Adib M, Ibáñez M, et al. La violencia obstétrica: un fenómeno vinculado a la violación de los derechos elementales de la mujer. Med Leg Costa Rica [internet]. 2017 [citado 2020 abr. 20]; 34(1):1-8. Disponible en: https:/www.scielo.sa.cr/pdf/mlcr/ v34n1/2215-5287-mlcr-34-01-104.pdf

4. México. Coordinación para la Atención de los Derechos Humanos (CADH) del Poder Ejecutivo del Estado de Oaxaca. Violencia obstétrica: violación a los derechos de las mujeres. Oaxaca de Juárez: CADH [internet]. 2018 [citado 2020 abr. 20]. Disponible en: https:/www.oaxaca.gob.mx/sinfra/wp-content/uploads/sites/14/2019/02/Violencia_obstetrica.pdf

5. Belli LF. La violencia obstétrica : otra forma de violación a los derechos humanos. Rev Redbioética/Unesco. 2013;1(7):25-34.

6. Correa ML. La humanización de la atención en los servicios de salud: un asunto de cuidado. Rev Cuid. 2016;7(1):1227-31. Dor: http://dx.doi.org/10.15649/cuidarte.v7i1.300

7. Barbas Á. Educomunicación: desarrollo, enfoques y desafíos en un mundo interconectado. Foro Educ. 2012;(14):157-75.

8. Cáceres-Manrique FM, Nieves-Cuervo GM, Lizarazo-Castellanos AD. Elaboración y validación de una cartilla sobre parto humanizado. Enferm Global. 2020;19(4):64-84. DoI: https://doi. org/10.6018/eglobal.412771

9. Piedrahita B. Diseño de una estrategia educomunicativa a través de un proceso de aprendizaje colaborativo secuenciado, que permita el desarrollo de la autonomía en la producción de contenidos audiovisuales, en espacios mediático-tecnológicos como lo son los magazines Enfokados y Ciudad 45. [Tesis doctoral]. Universidad Tecnológica de Pereira [internet]. 2016 [citado 2020 sep. 10]. Disponible en: http://repositorio.utp.edu.co/dspace/bitstream/ handle/11059/7357/3711022P613.pdf?sequence $=1$

10. Sánchez A, Salinas W. Estrategias educomunicativas para la formación de comportamientos ambientales responsables en escenarios empresariales: síntesis de la experiencia en la planta de Coca Cola, sede Bucaramanga. Rev Docencia e Investig. 2016;6(2):6577. DoI: https://doi.org/10.15332/erdi.v6i2.1650

11. Rodríguez-Triana JO. Estrategia educomunicativa para el fortalecimiento de la cultura ambiental en torno al manejo de residuos sólidos urbanos en la ciudad de Ibagué. [Tesis doctoral]. Ibagué: Universidad Nacional Abierta y a Distancia [internet]; 2018 [citado 2020 nov. 10]. Disponible en: https://repository.unad.edu.co/ jspui/bitstream/10596/17928/3/19202142.pdf

12. Galindo-Neto NM, Silva A, Moreira L, Guarino G, Mesquita K, Afio J. Construcción y validación de un vídeo educativo para sordos acerca de la reanimación cardiopulmonar. Rev Lat Am Enfermagem. 2019;27(e3130):1-12. DoI: http://dx.doi. org/10.1590/1518-8345.2765.3130
13. Pastor A, Melo C. Literature review of audiovisual practices in Nursing education. Rev Bras Enferm. 2019;72(1):190-9. DOI: http://dx.doi.org/10.1590/0034-7167-2017-0890

14. Campos-Câmara D. Elaboração e validação de vídeo educativo para prevenção de queda em criança hospitalizada. [Tesis doctoral]. Niterói: Universidade Federal Fluminense [internet]; 2019 [citado 2020 ago. 24]; 10(1):e0116380. https://app.uff.br/riuff/ handle/1/9424

15. Feo C, Feo O. Impacto de los medios de comunicación en la salud pública. Saúde em Debate. 2013;37(96):84-95. DoI: https://doi. org/10.1590/S0103-11042013000100010

16. Hidalgo J, Aliaga E. Análisis de las estrategias didácticas para el diseño, selección, producción, utilización y validación de recursos educativos audiovisuales interactivos en una institución educativa. Estudio inicial. Rev. Electron. Investig. Innov. Educ. 2020;(23):79-98. DOI: https://doi.org/10.17561/10.17561/reid. $\mathrm{n} 23.5$

17. Moquillaza Alcántara V, Romero Cerdán A. ¿Es posible impactar en la salud mediante redes sociales y podremos confiar en la información que trasmiten? Rev Int Salud Materno Fetal [internet]. 2019 [citado 2020 jul. 5]; 4(3):29-30. Disponible en: revistamaternofetal.com. ISSN 25199994

18. Espinoza-Portilla E, Mazuelos-Cardoza C. Desinformación sobre temas de salud en las redes sociales. Rev Cubana Inform Cienc Salud [internet]. 2020 [citado 2020 jul. 27] (31)2:e-1498. http:// www.rcics.sld.cu/index.php/acimed/article/view/1498

19. Ziemendorff S, Krause A. Guía de Validación de materiales educativos (con enfoque en materiales de educación sanitaria). Chiclayo: Programa de Agua y Saneamiento PROAGUA/ GTZ [internet]. 2003. [citado 2020 ago. 30]. Disponible en: https://www.researchgate.net/publication/33551746_Guia_ de_validacion_de_materiales_educativos_con_enfoque_en_materiales_de_educacion_sanitaria

20. Colombia. Ministerio de Salud y Protección Social. Guías de práctica clínica para la prevención, detección temprana y tratamiento de las complicaciones del embarazo, parto o puerperio. Guías N. ${ }^{\circ}$ 11-15 [internet]. 2013 [citado 2020 nov. 12]. Disponible en: https://www.minsalud.gov.co/sites/rid/Lists/BibliotecaDigital/RIDE/INEC/IETS/G.Corta.Embarazo.y.parto.Prof.Salud. $2013 \% 20(1) . p d f$

21. Technical Working Group, World Health Organization. Care in normal birth: A practical guide. Birth. [internet]. 1997 [citado 2021 ene. 4]; 24(2):121-3. Disponible en: https://onlinelibrary. wiley.com/doi/abs/10.1111/j.1523-536X.1997.00121.pp.x

22. Gértrudix M, Rajas M, Álvarez S. Metodología de producción para el desarrollo de contenidos audiovisuales y multimedia para MOOC. RIED. 2017;20(1):183-203. DOI: http://dx.doi.org/10.5944/ ried.20.1.16691

23. Gértrudix M, Rajas M, Barrera D, et al. Realización de vídeo educativo: análisis de la producción audiovisual de los MOOC de URJCx. En Sierra, J. Nuevas tecnologías audiovisuales para nuevas narrativas interactivas digitales en la era multidispositivo. Madrid: McGraw Hill Education; 2017. pp. 289-302.

24. Fehring RJ. The Fehring model. En: Carroll-Johnson RM, Paquette M, Classification of Nursing Diagnosis: Proceedings of the tenth conference. Philadelphia: JB Lippincott Company; 1994. pp. 55-7.

25. Fleiss JL, Cohen J, Everitt BS. Large sample standard errors of kappa and weighted kappa. Psychol Bull. 1969;72/(5):323-7. DoI: https://doi.org/10.1037/h0028106

26. República de Colombia. Ministerio de Salud. Resolución 8430 de 1993, por la cual se dictan normas para la investigación en 
seres humanos. Bogotá, Colombia [internet]. 1993 [citado 2020 nov. 10]. Disponible en: https://www.minsalud.gov.co/sites/rid/ Lists/BibliotecaDigital/RIDE/DE/DIJ/RESOLUCION-8430DE-1993.PDF

27. Santos-Silva AL, Nascimento ER do, Coelho-Cardozo EA. Nurses practices to promote dignity, participation and empowerment of women in natural childbirth. Esc Anna Nery [internet]. 2015 [citado 2020 sep. 30] 19(3):424-31. Disponible en: https://www. scielo.br/pdf/ean/v19n3/en_1414-8145-ean-19-03-0424.pdf
28. Díaz L, Fernández Y. Situación legislativa de la violencia obstétrica en América Latina: el caso de Venezuela, Argentina, México y Chile. Rev Derecho la Pontif Univ Católica Valparaíso. 2018;(51):123-44 DoI: http://dx.doi.org/10.4067/S071868512018005000301

29. Nascimento FCV, Silva MP, Viana MRP. Assistência de enfermagem no parto humanizado. Rev Pre Infec e Saúde. 2018;4:6887. DOI: https://doi.org/10.26694/repis.v4i0.6821 